\title{
Phosphane-free Suzuki-Miyaura Coupling of Aryl Imidazolesulfonates with Arylboronic Acids and Potassium Aryltrifluoroborates under Aqueous Conditions ${ }^{\#}$
}

\author{
José Francisco Cívicos, Mohammad Gholinejad, Diego A. Alonso,* and Carmen Nájera* \\ Departamento de Quimica Orgánica and Instituto de Síntesis Orgánica, Universidad de Alicante, \\ Apdo. 99, E-03080, Alicante, Spain
}

(Received March 8, 2011; CL-110199; E-mail: diego.alonso@ua.es, cnajera@ua.es)

Aryl imidazole-1-sulfonates are efficiently cross-coupled with arylboronic acids and potassium aryltrifluoroborates using only $0.5 \mathrm{~mol} \%$ of oxime palladacycles 1 under aqueous conditions at $110^{\circ} \mathrm{C}$. Under these simple phosphane-free reaction conditions a wide array of biaryl derivatives has been prepared in high yields. This methodology allows in situ phenol sulfonation and one-pot Suzuki arylation as well as the employment of microwave irradiation conditions.

The Suzuki-Miyaura reaction constitutes one of the most powerful carbon-carbon bond-forming transformations. ${ }^{1}$ Due to the broad existence of $O$-containing organic compounds in nature there has been a tremendous interest on the transitionmetal-catalyzed cross-coupling reactions of this type of compounds. ${ }^{2}$ The palladium-catalyzed Suzuki-Miyaura cross-coupling reaction of enols, phenols, and hydroxylated arenes usually involves their transformation into triflates due to the superior performance of these derivatives as electrophilic partners. ${ }^{3}$ However, triflates are substrates with limited stability. Thus, different studies have shown the ability of other functional groups, such as mesylates, ${ }^{4}$ tosylates, ${ }^{4 \mathrm{~d}, 5}$ carbamates, ${ }^{6}$ carbonates, ${ }^{6 \mathrm{a}}$ carboxylates, ${ }^{4 \mathrm{~b}, 7}$ ethers, ${ }^{8}$ phosphonium salts, ${ }^{9}$ phosphoramides, ${ }^{10}$ and $N, N$-dialkyl $O$-sulfamates, ${ }^{6 a, 11}$ to perform in the Suzuki reaction usually under palladium or nickel catalysis in the presence of phosphane ligands. Very recently, aryl imidazolesulfonates have been demonstrated as efficient electrophilic coupling partners in the Suzuki reaction with aryl boronic acids employing bidentate phosphane ligands, such as $\operatorname{dppf}\left[1,1^{\prime}\right.$-bis(diphenylphosphano)ferrocene] and BINAP, under high catalyst loadings $(5-10 \mathrm{~mol} \% \mathrm{Pd}) .^{11-13}$

In our research group, the Suzuki-Miyaura reaction has been studied during the past decade using oxime palladacycles 1 (Figure 1) as efficient precatalysts for the coupling of vinyl- and aryl halides with boronic acids and potassium trifluoroborates in organic and aqueous solvents. ${ }^{14,15}$ Herein we report the use of imidazolesulfonates as effective electrophilic coupling reagents in a phosphane-free Suzuki-Miyaura reaction with boronic acids<smiles>[R2]c1ccc2c(c1)[R](Cl)(Cl)N(O)C2[R]</smiles>

1a, $\mathrm{R}^{1}=\mathrm{Me}, \mathrm{R}^{2}=\mathrm{OH}$

1b, $\mathrm{R}^{1}=4-\mathrm{ClC}_{6} \mathrm{H}_{4}, \mathrm{R}^{2}=\mathrm{Cl}$

Figure 1. Oxime palladacycles. and potassium trifluoroborates catalyzed by oxime palladacycles 1 under aqueous conditions.

Initial Suzuki cross-coupling studies were carried out over 1-naphthol derivatives 2 (Table 1). The cross-coupling of compounds 2 ( 1 equiv) with phenylboronic acid (1.5 equiv) was initially performed using catalyst $\mathbf{1 a}^{16}(1 \mathrm{~mol} \% \mathrm{Pd})$ and $\mathrm{KOH}$ ( 2 equiv) as base in a mixture $\mathrm{MeOH} / \mathrm{H}_{2} \mathrm{O}: 3 / 1$ as solvent in the presence of tetrabutylammonium bromide (TBAB) $(20 \mathrm{~mol} \%)$ as phase transfer agent at $110^{\circ} \mathrm{C}$ (Table 1, Entries 1-5). As depicted in Table 1, only the imidazolesulfonate derivative 2ae showed reactivity in the process affording 1-phenylnaphthalene (3a) in a $67 \%$ isolated yield.

Once demonstrated that the imidazole-1-sulfonate was the best electrophile in the process, an optimization of the reaction

Table 1. Reaction conditions study ${ }^{\mathrm{a}}$<smiles>[R]c1cccc2ccccc12</smiles>

2aa, $\mathrm{R}=\mathrm{CO}_{2} t$-Bu

2ab, $\mathrm{R}=\mathrm{CONMe}$

2ac, $\mathrm{R}=\mathrm{P}(\mathrm{O})(2-\mathrm{oxooxazolidin-3-yl)}$

2ad, $\mathrm{R}=\mathrm{SO}_{2} \mathrm{NMe}_{2}$

2ae, $\mathrm{R}=\mathrm{SO}_{2}$ (imidazol-1-yl)

\begin{tabular}{cllllc}
\hline Entry & $\mathbf{2}$ & $\mathrm{BX}$ & Catalyst & Solvent $^{\mathrm{b}}$ & ${\text { Yield } / \%^{\mathrm{c}}}^{\mathrm{a}}$ \\
\hline 1 & $\mathbf{2 a a}$ & $\mathrm{B}(\mathrm{OH})_{2}$ & $\mathbf{1 a}$ & $\mathrm{A}$ & $<5$ \\
2 & $\mathbf{2 a b}$ & $\mathrm{B}(\mathrm{OH})_{2}$ & $\mathbf{1 a}$ & $\mathrm{A}$ & $<5$ \\
3 & $\mathbf{2 a c}$ & $\mathrm{B}(\mathrm{OH})_{2}$ & $\mathbf{1 a}$ & $\mathrm{A}$ & $<5$ \\
4 & $\mathbf{2 a d}$ & $\mathrm{B}(\mathrm{OH})_{2}$ & $\mathbf{1 a}$ & $\mathrm{A}$ & $<5$ \\
5 & $\mathbf{2 a e}$ & $\mathrm{B}(\mathrm{OH})_{2}$ & $\mathbf{1 a}$ & $\mathrm{A}$ & 67 \\
6 & $\mathbf{2 a e}$ & $\mathrm{B}(\mathrm{OH})_{2}$ & $\mathbf{1 a}$ & $\mathrm{B}$ & $<5$ \\
7 & $\mathbf{2 a e}$ & $\mathrm{B}(\mathrm{OH})_{2}$ & $\mathbf{1 a}$ & $\mathrm{H}_{2} \mathrm{O}$ & $<5$ \\
8 & $\mathbf{2 a e}$ & $\mathrm{B}(\mathrm{OH})_{2}$ & $\mathrm{Pd}(\mathrm{OAc})_{2}$ & $\mathrm{~A}$ & 84 \\
9 & $\mathbf{2 a e}$ & $\mathrm{B}(\mathrm{OH})_{2}$ & $\mathbf{1 b}$ & $\mathrm{A}$ & $>99$ \\
10 & $\mathbf{2 a e}$ & $\mathrm{B}(\mathrm{OH})_{2}$ & $\mathbf{1 b}$ & $\mathrm{A}$ & $>99^{\mathrm{d}}$ \\
11 & $\mathbf{2 a e}$ & $\mathrm{BF} \mathrm{K}_{3}$ & $\mathbf{1 a}$ & $\mathrm{A}$ & 89 \\
12 & $\mathbf{2 a e}$ & $\mathrm{BF}_{3} \mathrm{~K}$ & $\mathbf{1 b}$ & $\mathrm{A}$ & 45 \\
13 & $\mathbf{2 a e}$ & $\mathrm{BF}_{3} \mathrm{~K}$ & $\mathrm{Pd}(\mathrm{OAc})_{2}$ & $\mathrm{~A}$ & 10 \\
14 & $\mathbf{2 a e}$ & $\mathrm{BX}^{\mathrm{e}}$ & $\mathbf{1 a}$ & $\mathrm{A}$ & $50^{\mathrm{f}}$ \\
15 & $\mathbf{2 a e}$ & $\mathrm{BX}^{\mathrm{g}}$ & $\mathbf{1 b}$ & $\mathrm{A}$ & $39^{\mathrm{f}}$ \\
\hline
\end{tabular}

aReaction conditions: Pd catalyst ( $1 \mathrm{~mol} \% \mathrm{Pd}), 2(0.1 \mathrm{mmol})$, PhBX (0.15 mmol), TBAB (20 mol \%), KOH (0.2 mmol) under A or B solvent conditions. ${ }^{\mathrm{b}} \mathrm{A}: \mathrm{MeOH} / \mathrm{H}_{2} \mathrm{O}: 3 / 1 ; \mathrm{B}: \mathrm{Me}_{2} \mathrm{CO} /$ $\mathrm{H}_{2} \mathrm{O}: 3 / 2$. ${ }^{\mathrm{c}}$ Isolated yield after flash chromatography. ${ }^{\mathrm{d}}$ Reaction performed under microwave irradiation conditions $(40 \mathrm{~W}$, $\left.110{ }^{\circ} \mathrm{C}, 30 \mathrm{~min}\right) .{ }^{\mathrm{e}} \mathrm{BX}=\mathrm{B}\left[\left(\mathrm{OCOCH}_{2}\right)_{2} \mathrm{NMe}\right] .{ }^{\mathrm{f}}$ Reaction time: 2 days. ${ }^{\mathrm{g}} \mathrm{BX}=\mathrm{B}\left[\left(\mathrm{OC}(\mathrm{Me})_{2}\right)\right]_{2}$. 
Table 2. Substrate scope

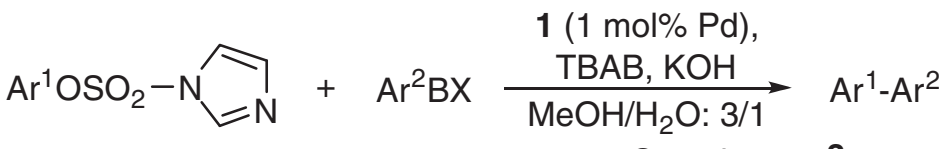

$$
\begin{aligned}
& 110^{\circ} \mathrm{C}, 24 \mathrm{~h}
\end{aligned}
$$

\begin{tabular}{|c|c|c|c|c|c|c|}
\hline \multirow{2}{*}{ Entry } & \multirow{2}{*}{$\mathrm{Ar}^{1} \mathrm{OSO}_{2} \mathrm{Im}$} & \multirow{2}{*}{ No. } & \multirow{2}{*}{$\mathrm{Ar}^{2} \mathrm{BX}$} & \multirow{2}{*}{ Palladacycle } & \multicolumn{2}{|c|}{ Product } \\
\hline & & & & & No. & Yield $/ \%^{a}$ \\
\hline 1 & & $2 a e$ & $4-\mathrm{MeC}_{6} \mathrm{H}_{4} \mathrm{~B}(\mathrm{OH})_{2}$ & $1 a$ & $3 b$ & 90 \\
\hline 2 & & $2 a e$ & $4-\mathrm{MeC}_{6} \mathrm{H}_{4} \mathrm{~B}(\mathrm{OH})_{2}$ & $1 b$ & $3 b$ & 76 \\
\hline 3 & & $2 \mathrm{ae}$ & $4-\mathrm{MeC}_{6} \mathrm{H}_{4} \mathrm{BF}_{3} \mathrm{~K}$ & $1 a$ & $3 b$ & 61 \\
\hline 4 & & $2 \mathrm{ae}$ & 4- $\mathrm{MeC}_{6} \mathrm{H}_{4} \mathrm{BF}_{3} \mathrm{~K}$ & $1 b$ & $3 b$ & 72 \\
\hline 5 & & $2 \mathrm{ae}$ & $4-\mathrm{MeOC}_{6} \mathrm{H}_{4} \mathrm{~B}(\mathrm{OH})_{2}$ & $1 \mathrm{~b}$ & $3 c$ & 75 \\
\hline 6 & & $2 \mathrm{ae}$ & $4-\mathrm{CF}_{3} \mathrm{C}_{6} \mathrm{H}_{4} \mathrm{~B}(\mathrm{OH})_{2}$ & $1 \mathrm{~b}$ & $3 d$ & 66 \\
\hline 7 & & $2 a e$ & $4-\mathrm{CF}_{3} \mathrm{C}_{6} \mathrm{H}_{4} \mathrm{BF}_{3} \mathrm{~K}$ & $1 \mathrm{~b}$ & $3 d$ & 64 \\
\hline 8 & & $2 b e$ & 4- $\mathrm{MeC}_{6} \mathrm{H}_{4} \mathrm{~B}(\mathrm{OH})_{2}$ & $1 \mathrm{~b}$ & $3 e$ & 86 \\
\hline 9 & & $2 \mathrm{ce}$ & $\mathrm{PhB}(\mathrm{OH})_{2}$ & $1 \mathrm{~b}$ & $3 e$ & 90 \\
\hline 10 & & $2 \mathrm{de}$ & $\mathrm{PhB}(\mathrm{OH})_{2}$ & $1 \mathrm{~b}$ & $3 f$ & 85 \\
\hline 11 & & $2 e e$ & $\mathrm{PhB}(\mathrm{OH})_{2}$ & $1 b$ & $3 g$ & 81 \\
\hline 12 & & $2 \mathrm{fe}$ & $\mathrm{PhB}(\mathrm{OH})_{2}$ & $1 b$ & $3 h$ & 78 \\
\hline 13 & & $2 \mathrm{ge}$ & $\mathrm{PhB}(\mathrm{OH})_{2}$ & $1 b$ & $3 \mathbf{i}$ & 53 \\
\hline
\end{tabular}

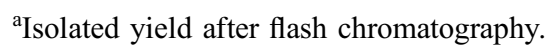

conditions was carried out. No reaction took place when the coupling was performed in aqueous acetone or in neat water (Table 1, Entries 6 and 7). Regarding catalysts, $\mathrm{Pd}(\mathrm{OAc})_{2}$ afforded a higher (84\%) yield in the reaction (Table 1, compare Entries 5 and 8). To our surprise, the yield improved substantially $(99 \%)$ when palladacycle $\mathbf{1 b}$, catalyst which usually performs better in organic solvents, ${ }^{15}$ was used (Table 1 , Entry 9). In the presence of catalyst $\mathbf{1 b}$ the reaction could also be performed under microwave irradiation conditions $(40 \mathrm{~W}$, $110^{\circ} \mathrm{C}$ ), affording compound $\mathbf{3 a}$ in quantitative yield after only $30 \mathrm{~min}$ (Table 1, Entry 10). The optimized results obtained with different boron electrophiles are shown in Entries 11-15. Potassium phenyltrifluoroborate afforded compound 3a in a good $89 \%$ isolated yield using catalyst 1a (Table 1, Entry 11).
With this nucleophile, catalyst $\mathbf{1 b}$ and $\mathrm{Pd}(\mathrm{OAc})_{2}$ were much less reactive than 1a affording compound $\mathbf{3 a}$ in a poor 45 and $10 \%$ yield, respectively (Table 1, Entries 12 and 13). Low yields were also obtained after two days when imidazolesulfonate 2 ae reacted with phenylboronic acid MIDA ester (50\%, Table 1, Entry 14) and phenylboronic acid pinacol ester (39\%, Table 1, Entry 15).

The scope of this methodology was then examined under the optimized reaction conditions. Cross-coupling of naphthalen1-yl $1 H$-imidazole-1-sulfonate (2ae) with diverse arylboronic acids and potassium trifluoroborates, afforded the corresponding biaryl derivatives $\mathbf{3 b}$-3d in good to excellent yields (Table 2, Entries 1-7). Generally better yields were observed for boronic acids than for potassium trifluoroborates being the lowest yields, 


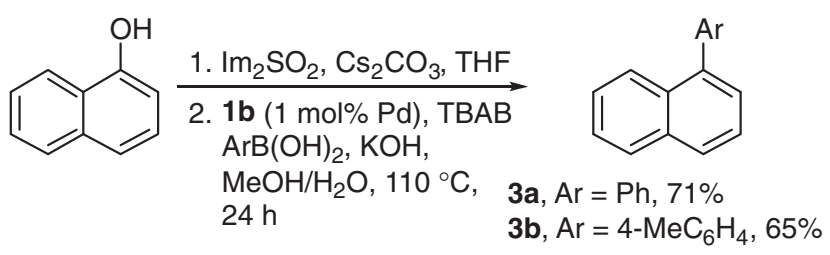

Scheme 1. One-pot sulfonation/cross-coupling sequence.

those obtained when electron-deficient nucleophiles were used (Table 2, Entries 6 and 7). Also, catalyst 1b performed usually better than 1a, though no clear correlation between the catalyst and the boron source could be established from parallel experiments. Thus, further studies on the reaction scope and utility of the method were always optimized with respect to the catalyst, the best results being shown in Table 2 . Regarding the electrophilic component (Table 2, Entries 8-13), high isolated yields (81-90\%) were obtained in the 1b-catalyzed crosscoupling of arylboronic acids with neutral, electron-rich, and electron-poor phenyl imidazolesulfonates (Table 2, Entries 811). The process was also effective for the coupling of sterically hindered electrophiles such as 2fe and 2ge (Table 2, Entries 12 and 13 ), though a moderate $53 \%$ isolated yield was observed for the more sterically demanding derivative 2 ge.

As the synthesis of imidazolesulfonates typically proceeds quantitatively and with minimal by-product formation, we carried out a one-pot sulfonation/Suzuki cross-coupling sequence of 1-naphthol with phenyl- and 4-tolylboronic acids. As depicted in Scheme 1, the one-pot conversion of 1-naphthol to biaryl adducts $\mathbf{3 a}$ and $\mathbf{3 b}$ was performed by reaction of 1-naphthol with $1,1^{\prime}$-sulfonyldiimidazole in THF using cesium carbonate as base and subsequent 1b-catalyzed Suzuki crosscoupling of the in situ generated imidazole-1-sulfonate with the corresponding arylboronic acid, to afford the desired products in a $71 \%$ and $65 \%$ isolated yield, respectively.

In conclusion, we have disclosed a general phosphane-free oxime-palladacycle-catalyzed Suzuki cross-coupling reaction of electron-rich, electron-poor, and hindered aryl imidazolesulfonates with arylboronic acids and potassium aryltrifluoroborates under aqueous conditions. The reaction, which can be performed under conventional heating or microwave irradiation, has been optimized for use with only $1 \mathrm{~mol} \% \mathrm{Pd}$ of bench stable palladacycles 1. Furthermore, this methodology allows in situ phenol sulfonation and one-pot Suzuki arylation. Currently, further studies are underway addressing extension of this methodology to other palladium-catalyzed transformations. $^{17}$

Financial support from the MICINN (Projects CTQ200762771/BQU, CTQ2010-20387, and Consolider INGENIO 2010 CSD2007-00006), FEDER, the Generalitat Valenciana (Projects GV/2007/142 and PROMETEO/2009/038), and the University of Alicante is acknowledged. M. G. thanks Shiraz University and University of Alicante for grants.

This paper is in celebration of the 2010 Nobel Prize awarded to Professors Richard F. Heck, Akira Suzuki, and Ei-ichi Negishi.
References and Notes

\# Dedicated to Prof. Alfredo Ricci on the occasion of his retirement.

1 a) N. Miyaura, A. Suzuki, Chem. Rev. 1995, 95, 2457. b) S. Kotha, K. Lahiri, D. Kashinath, Tetrahedron 2002, 58, 9633. c) A. F. Littke, G. C. Fu, Angew. Chem., Int. Ed. 2002, 41, 4176. d) F. Bellina, A. Carpita, R. Rossi, Synthesis 2004, 2419. e) U. Christmann, R. Vilar, Angew. Chem., Int. Ed. 2005, 44, 366. f) F. Alonso, I. P. Beletskaya, M. Yus, Tetrahedron 2008, 64, 3047. g) Metal-Catalyzed Cross-Coupling Reactions, ed. by A. de Meijere, F. Diederich, Wiley-VCH, Weinheim, 2004, Vol. 2.

2 For recent reviews on the use of new $\mathrm{C}-\mathrm{O}$ electrophiles in crosscoupling reactions, see: a) D.-G. Yu, B.-J. Li, Z.-J. Shi, Acc. Chem. Res. 2010, 43, 1486. b) B.-J. Li, D.-G. Yu, C.-L. Sun, Z.-J. Shi, Chem.-Eur. J. 2011, 17, 1728.

3 a) W. J. Scott, G. T. Crisp, J. K. Stille, J. Am. Chem. Soc. 1984, 106, 4630. b) G. Zeni, R. C. Larock, Chem. Rev. 2006, 106, 4644.

4 For recent examples, see: a) C. M. So, C. P. Lau, F. Y. Kwong, Angew. Chem., Int. Ed. 2008, 47, 8059. b) G. A. Molander, F. Beaumard, Org. Lett. 2010, 12, 4022. c) W. K. Chow, C. M. So, C. P. Lau, F. Y. Kwong, J. Org. Chem. 2010, 75, 5109. d) H. Gao, Y. Li, Y.-G. Zhou, F.-S. Han, Y.-J. Lin, Adv. Synth. Catal. 2011, 353, 309.

5 For recent examples, see: a) J. P. Wolfe, H. Tomori, J. P. Sadighi, J. Yin, S. L. Buchwald, J. Org. Chem. 2000, 65, 1158. b) D.-Y. Lee, J. F. Hartwig, Org. Lett. 2005, 7, 1169. c) L. Zhang, T. Meng, J. Wu, J. Org. Chem. 2007, 72, 9346.

6 a) K. W. Quasdorf, M. Riener, K. V. Petrova, N. K. Garg, J. Am. Chem. Soc. 2009, 131, 17748. b) A. Antoft-Finch, T. Blackburn, V. Snieckus, J. Am. Chem. Soc. 2009, 131, 17750. c) L. Xu, B.-J. Li, Z.-H. Wu, X.-Y. Lu, B.-T. Guan, B.-Q. Wang, K.-Q. Zhao, Z.-J. Shi, Org. Lett. 2010, 12, 884.

7 a) B.-T. Guan, Y. Wang, B.-J. Li, D.-G. Yu, Z.-J. Shi, J. Am. Chem. Soc. 2008, 130, 14468. b) K. W. Quasdorf, X. Tian, N. K. Garg, J. Am. Chem. Soc. 2008, 130, 14422. c) C.-L. Sun, Y. Wang, X. Zhou, Z.-H. Wu, B.-J. Li, B.-T. Guan, Z.-J. Shi, Chem.-Eur. J. 2010, 16, 5844.

8 For a recent example, see: M. Tobisu, T. Shimasaki, N. Chatani, Angew. Chem., Int. Ed. 2008, 47, 4866.

9 a) F.-A. Kang, Z. Sui, W. V. Murray, J. Am. Chem. Soc. 2008, 130, 11300. b) F.-A. Kang, Z. Sui, W. V. Murray, Eur. J. Org. Chem. 2009, 461.

10 Y.-L. Zhao, Y. Li, Y. Li, L.-X. Gao, F.-S. Han, Chem.-Eur. J. 2010, 16, 4991.

11 T. K. Macklin, V. Snieckus, Org. Lett. 2005, 7, 2519.

12 J. Albaneze-Walker, R. Raju, J. A. Vance, A. J. Goodman, M. R. Reeder, J. Liao, M. T. Maust, P. A. Irish, P. Espino, D. R. Andrews, Org. Lett. 2009, 11, 1463.

13 For the use of aryl imidazolesulfonates in other cross-coupling reactions, see: a) Y. Luo, J. Wu, Organometallics 2009, 28, 6823. b) S. J. Shirbin, B. A. Boughton, S. C. Zammit, S. D. Zanatta, S. M. Marcuccio, C. A. Hutton, S. J. Williams, Tetrahedron Lett. 2010, 51, 2971. c) L. Ackermann, S. Barfüsser, J. Pospech, Org. Lett. 2010, 12, 724.

14 Palladacycles: Synthesis, Characterization and Applications, ed. by J. Dupont, M. Pfeffer, Wiley-VCH, Weinheim, 2008.

15 For recent reviews on the use of oxime-palladacycles as precatalysts in cross-coupling reactions, see: a) E. Alacid, D. A. Alonso, L. Botella, C. Nájera, M. C. Pacheco, Chem. Rec. 2006, 6, 117. b) D. A. Alonso, C. Nájera, Chem. Soc. Rev. 2010, 39, 2891.

16 Catalyst 1a has shown to be very effective for cross-coupling and Heck reactions under aqueous conditions. See ref. 15.

17 Supporting Information is available electronically on the CSJJournal Web site, http://www.csj.jp/journals/chem-lett/index.html. 